\title{
MODELLING COVID-19 PANDEMIC IN NIGERIA USING MULTIVARIATE AUTOREGRESSIVE DISTRIBUTED LAG-MOVING AVERAGE MODELS
}

\section{Usoro Anthony E. and John Eme E.}

Department of Statistics, Akwa Ibom State University, Mkpat Enin, Akwa Ibom State, Nigeria.

Cite this article:

Usoro A.E., John E.E. (2021), Modelling Covid-19

Pandemic in Nigeria using Multivariate Autoregressive Distributed Lag-Moving Average Models. African Journal of Mathematics and Statistics Studies 4(3), 118134. DOI: $10.52589 / A J M S S-$ VFKTCGIK.

\section{Manuscript History}

Received: 7 Oct 2021

Accepted: 23 Oct 2021

Published: 19 Nov 2021

Copyright $(92020$ The Author(s). This is an Open Access article distributed under the terms of Creative Commons AttributionNonCommercial-NoDerivatives 4.0 International (CC BY-NC-ND 4.0 ), which permits anyone to share, use, reproduce and redistribute in any medium, provided the original author and source are credited.

\begin{abstract}
The aim of this paper was to study the trend of COVID-19 cases and fit appropriate multivariate time series models as research to complement the clinical and non-clinical measures against the menace. The cases of COVID-19, as reported by the National Centre for Disease Control (NCDC) on a daily and weekly basis, include Total Cases (TC), New Cases (NC), Active Cases (AC), Discharged Cases (DC) and Total Deaths (TD). The three waves of the COVID-19 pandemic are graphically represented in the various time plots, indicating the peaks as (June-August, 2020), (December-February, 2021), and (July-September, 2021). Multivariate Autoregressive Distributed Lag Models (MARDLM) and Multivariate Autoregressive Distributed Lag Moving Average (MARDL-MA) models have been found to be suitable for fitting different categories of the COVID-19 pandemic in Nigeria. The graphical representation and estimates have shown a gradual decline in the reported cases after the peak in September 2021. So far, the introduction of vaccines and non-pharmaceutical measures by relevant organisations are yielding plausible results, as evident in the recent decrease in New Cases, Active Cases and an increasing number of Discharged Cases, with fewer deaths. This paper advocates consistency in all clinical and non-clinical measures as a way towards the extinction of the dreaded COVID-19 pandemic in Nigeria and the world.
\end{abstract}

KEYWORDS: COVID-19, MARDLM, MARDL-MA. 


\section{INTRODUCTION}

Coronavirus (COVID-19) is an infectious disease caused by severe acute respiratory syndrome coronavirus 2 (SARS-COVID-2). According to the World Health Organisation (WHO), coronavirus (COVID-19) is a newly identified virus that has caused monumental effects and has subjected the world to a health disaster, as no continent on the planet earth is free from the life-threatening and tragic pandemic. The entire human race is currently facing a tremendous health crisis with the rapid spread of the disease throughout the world. The infectious disease was first identified in December, 2019 in Wuhan, China. The countries with high vulnerability at the early stage of the outbreak were China, Spain, Italy, the USA, and the UK. As a temperate region, its effect on the African continent was not alarming as only a few cases were reported at the early stage of the outbreak.

In Africa, Nigeria recorded the first case of the coronavirus pandemic on February 27, 2020, from an Italian man who was diagnosed with the infectious disease in a laboratory in Lagos. Following the trend of the pandemic in other countries, the incidence triggered fear and reactions that the virus could be disastrous if proactive measures were not taken by the government to curb its spread (Maclean \& Abdi, 2020). As part of the measures to prevent the spread of the disease in Nigeria, the federal government expressed commitment to surveillance at five international airports in Nigeria. The five airports were Enugu, Kano, Lagos, Rivers, and the FCT. This was followed by the inter-multisectoral committee which comprised stakeholders in health, security, aviation, and transport sectors as a build up for surveillance to forestall the introduction of coronavirus by foreigners and Nigerians from abroad (Oladeinde, 2020). The federal government, through the Minister of Health, Prof. Osagie Ehanire, advocated strong collaborative efforts between the relevant national and international health agencies - the Nigeria Centre for Disease Control (NCDC) and the World Health Organisation (WHO) - to strengthen surveillance. This also included the states hosting international airports. Governments at the state level equally exhibited proactive measures, especially at local airports and inter-state borders, to check the importation of the infectious disease from one state to the other. To cushion the effects of the pandemic, federal and state governments provided palliatives to vulnerable people whose means of livelihood were affected by the total lockdown directive of the federal and state governments in a bid to avert the spread of the pandemic.

To further strengthen the fight against the spread of the disease, the World Health Organization (WHO) advised national, state, and local relevant health authorities to carry out public education advocacy and campaigns against the disease through the introduction of health evasive measures, which include: (i) regular washing of hands with soap; (ii) rubbing alcoholbased hand sanitizer on hand; (iii) maintaining social distance of a minimum of 1 meter or 3 feet from someone who coughs or sneezes. The proposed measures to mitigate the rapid spread of the COVID-19 pandemic were prompted as the World Health Organisation (WHO) identified Nigeria among the 13 African countries at high risk of coronavirus. Included with Nigeria were Algeria, Angola, Ivory Coast, DR. Congo, Ethiopia, Ghana, Kenya, Mauritius, South Africa, Tanzania, Uganda and Zambia. The reason was attributed to the frequent travel of the aforementioned countries to China, which may be due to the bilateral economic relations the countries have with China and individual business transactions. Despite the efforts and trend of government activities to prevent the scourge of the coronavirus pandemic in the country, Nigeria was among the first five countries on the continent that were mostly affected by the disease. This was very worrisome and called for concern by governments, stakeholders, and individuals in society. The weekly figures published by NCDC as at September 26, 2021 
recorded 204,456 Total Cases, 2,658 New Cases, 9,157 Active Cases, 192,622 Discharged Cases and 2,677 Total Deaths in Nigeria.

\section{COVID-19's Economic Impact}

The adverse effect of the pandemic cuts across many sectors including agriculture, oil and gas, education, services, mining, energy, and health as the worst hit sectors due to overstretching of manpower, facilities, and resources. The high risk of infection in the health sector instills fear in medical personnel, some of whom occasionally renege and break commitments in their responsibilities, failing to provide the expected services while some patients are left to their fates. Apart from the challenges in the health sector, the huge impact of the pandemic on oil and gas is very worrisome in view of the country's high reliance and dependence on oil as the major contributor to the economic growth of the nation. An instance is the envisaged negative effects on multinational companies in the era of this health crisis. This is evident as the Managing Director of Shell Nigeria Gas (SNG) Ltd expressed fears of an overwhelming economic downturn following the shutdown of its 118 customers during the peak of the lockdown. In addition to the shortfalls in the international market price of crude oil, this compelled the company to scale down its operations, reducing its production capacity (Premium Times; August 18, 2020). The negative impact of COVID-19 as a result of low turnout in production has affected the obligations of oil companies to the government and host communities in the areas of right of royalties, manpower development, and intervention in some basic amenities and infrastructure to promote economic diversification and stability. As many companies, industries, and many private organisations closed their doors during the peak of COVID-19, it resulted in an increase in the rate of unemployment across states because of the downsizing of the labor force, which is a major driving force of every nation's economy. Many businesses involved in the production of goods and services were forced to close their doors, particularly those that are not technologically advanced enough to necessitate working from home.

\section{REVIEW OF RELATED LITERATURE}

A little background literature on COVID-19 is built on the online publications and imprints on the statistics of the globally recorded cases. Despite the short period of discovery, a number of research in mathematical sciences have been established, including clinical trials for the discovery of a vaccine against the disease. This paper reviews related works published in COVID-19. Enahoro et al. (2020) adopted the Kermack-Mckendrick-Type compartmental epidemic model for the modelling and analysis of the COVID-19 pandemic in Nigeria. This model is a deterministic system of nonlinear differential equations and it reveals disease-free equilibria, which can be controlled whenever a certain epidemiological threshold has a value less than unity. Enahoro et al. (2020) split the human population at time t into mutually exclusive compartments of susceptible population, exposed population, symptoticallyinfectious population, asymptotically-infectious population, hospitalized population, and recovered population. The major focus of Enahoro et al. (2020) was on the effect of nonpharmaceutical intervention on COVID-19 prevalence in Nigeria, with the aim of considering the appropriate time it would be safe to relax the lockdown without exposing the human population to the risk of a second wave of the disease aside from model building, with the view of proposing a convenient and safer time to reopen the economy. Kayode et al. (2020) carried 
out a comparative analysis of models and estimators in the study of the COVID-19 pandemic in Nigeria. The paper viewed daily, cumulative, discharged, and dead cases. Their work identified the Quartic Linear Regression (QLR) model with an auto-correlated error of order 1, [AR(1)] and found OLS, Cochrane, Orcuh, Hildreth-Lu, Prais-Winsten, and Least Absolute Deviation estimators useful to estimate the parameters of the model. Vasilis (2020) carried out predictive modelling of COVID-19 data in the United States. An adaptive phase-space approach was adopted by dividing the population of study into susceptible, infectious, and recovered/removed fractions and defining their dynamic inter-relationships with first-order differential equations. The results of the work revealed a gradual reduction in the infectivity rate, although if adequate measures are not properly taken to control it, the latest waves of infection will be disastrous. Vini et al. (2020) adopted basic statistical inference to analyse COVID-19 data in Burkina Faso. The analysis involved point and interval estimations of average contamination and variance of distributions of COVID-19 cases in Burkina Faso. In Nigeria, COVID-19 cases are recorded in the categories: total cases, new cases, active cases, discharged cases, and total deaths. This gives reason to propose multivariate time series models for this work.

Johnston and Dinardo (1997) presented the general form of the univariate autoregressive distributed lag model ADL (p, q) in the form,

$$
z_{t}=\mu+\alpha_{1} z_{t-1}+\cdots+\alpha_{p} z_{t-p}+\beta_{0} y_{t}+\beta_{1} y_{t-1}+\cdots+\beta_{q} y_{t-q}+\epsilon_{t}
$$

where $z_{t}$ is the response time series variable, $y_{t}$ is the predictor time variable, $\mu$ is the universal mean and $\epsilon_{t}$ is the error term, $\epsilon_{t} \sim i i\left(0, \sigma_{\epsilon}^{2}\right)$. From the model, $z_{t}$ is a function of $z_{t-k(k=1, \ldots, p)}$ and $y_{t-k(k=0, \ldots, q)}$. Gujarati and Porter (2009) defined VAR models for Canadian money and interest rate as

$$
\begin{aligned}
& M_{t}=\mu+\sum_{i=1}^{k} \beta_{i} M_{t-i} \\
&+\sum_{i=1}^{k} \gamma_{i} R_{t-i} \\
& R_{t}=\varphi+\sum_{i=1}^{k} \begin{array}{l}
\pi_{i} M_{t-i} \\
+\sum_{i=1}^{k} \alpha_{i} R_{t-i}
\end{array}
\end{aligned}
$$

where $M_{t}$ and $R_{t}$ represent money and interest rate with associated parameters $\beta_{i}, \pi_{i}$ and $\gamma_{i}, \alpha_{i}$ respectively. Equation " 2 " is a set of bivariate time series models also known as VAR models. With these models, causality between the two response variables are established. 


\section{MODEL DERIVATION}

\section{Multivariate Autoregressive Distributed Lag Model}

\section{Proposition}

Let $X_{j t(j=1, \ldots, m)}$ be $m \times 1$ matrix of response variables, $\alpha_{i . j k(i=1, \ldots, p, j=1, \ldots, m, k=1, \ldots, n)}$ be a square matrix of coefficients, $C_{j(j=1, \ldots, m)}$ be $m \times 1$ matrix of constants and $\epsilon_{j t(j=1, \ldots, m)}$ be $m \times 1$ matrix of errors; $X_{k t}$ represents a vector of predictor variables, $j$ and $k$ are the subscripts for response and predictor variables respectively, while $m$ and $n$ are the numbers of response and predictor variables respectively. If $i=0(i \neq 1, \ldots, p)$ and $i \neq 0(i=1, \ldots, p)$, then, $X_{j t}$ is a linear combination of $X_{k t-i(k=1, \ldots, n, i=0,)}$ and $X_{k t-i(k=1, \ldots, n, i=1, \ldots, p)}$, and $X_{j t}$ is a Multivariate Autoregressive Distributed Lag Model in the form,

$$
X_{j t}=C_{j}+\sum_{\substack{i=0, i=1 \\+\epsilon_{j t}}}^{p} \sum_{j=1}^{m} \sum_{k=1}^{n} \alpha_{i . j k} X_{k t-i}
$$

The above model is the aggregation of Vector Regression Models (VRM) and Vector Autoregressive Models (VARM) (Usoro, 2019).

\section{Derivation:}

Given $X_{j}$ and $X_{k}$ to be two time series variables, with $\alpha_{i . j k}$ as the parameter of contribution of $X_{k}$ to $X_{j}$ at lag $i$, let $\alpha_{i . j k(j=k)}$ be associated with the lag polynomials,

$$
\left(1-\sum_{i=1}^{p} \quad \alpha_{i .11} L^{i}\right),\left(1-\sum_{i=1}^{p} \quad \alpha_{i .22} L^{i}\right), \ldots,\left(1-\sum_{i=1}^{p} \quad \alpha_{i . m n} L^{i}\right), \text { where } m=n
$$

and

$\alpha_{i . j k(j \neq k)}$, with the lag polynomials,

$$
\begin{gathered}
\left(-\sum_{i=1}^{p} \alpha_{i .11} L^{i}\right), \\
\text { for } j=1, k=2,3, \ldots, n \\
j=2, k=1,3, \ldots, n \\
j=3, k=1,2, \ldots, n \\
j=m, k=1,2,3, \ldots, n-1
\end{gathered}
$$


Let $\alpha(L)$ be a linear combination of

$$
\left(1-\sum_{i=1}^{p} \alpha_{i . j k(j=k)} L^{i}\right) \text { and }\left(-\sum_{i=1}^{p} \alpha_{i . j k(k \neq)} L^{i}\right),
$$

such that for each response variable,

$$
\begin{gathered}
\alpha_{1}(L)=\left(1-\sum_{\substack{i=1 \\
=0}}^{p} \alpha_{i .11} L^{i}\right)-\sum_{\substack{i=0 \\
(4)}}^{p} \alpha_{i .12} L^{i}-\sum_{i=0}^{p} \alpha_{i .13} L^{i}-\cdots-\sum_{i=0}^{p} \alpha_{i .1 n} L^{i} \\
\alpha_{2}(L)=-\sum_{i=0}^{p} \alpha_{i .21} L^{i}+\left(1-\sum_{i=1}^{p} \alpha_{i .22} L^{i}\right)-\sum_{i=0}^{p} \alpha_{i .23} L^{i}-\cdots-\sum_{i=0}^{p} \alpha_{i .2 n} L^{i} \\
\alpha_{m}(L)=-\sum_{i=0}^{p} \alpha_{i . m 1} L^{i}-\sum_{i=0}^{p} \alpha_{i . m 2} L^{i}-\sum_{i=0}^{p} \alpha_{i . m 3} L^{i}-\cdots+\left(1-\sum_{i=1}^{p} \alpha_{i . m n} L^{i}\right)
\end{gathered}
$$

The multiplications of (4), (5) and (6) by $X_{1 t}, X_{2 t}, \ldots, X_{m t}$ respectively produce Multivariate Autoregressive Distributed Lag Models, which are reduced to the form,

$$
X_{j t}=C_{j}+\sum_{\substack{i=0, i=1 \\+\epsilon_{j t}}}^{p} \sum_{j=1}^{m} \sum_{k=1}^{n} \alpha_{i . j k} X_{k t-i}
$$

where $X_{j t}$ is an $m \times 1$ vector matrix, $\alpha_{i . j k}(i=1, \ldots, \mathrm{p}, j=1, \ldots, \mathrm{m}, k=1, \ldots, n)$ are matrices of coefficients, $C_{j}$ is an $m \times 1$ vector of constants and $\epsilon_{j t}$ is an error term, $\epsilon_{j t} \sim i i d\left(0, \sigma_{\epsilon}^{2}\right)$. The difference between $X_{k t}$ and $X_{j t}$ is that each subscripted " $k$ " in $X_{k t}$ defines a contributor (predictor term) to each subscripted " $j$ " in $X_{j t}$, with the associated parameter $\alpha_{j k}$ indicating the contribution of " $k$ " to " $j "$.

From (7), if $i=0$, it defines Multi-Dependent Linear Regression Models (MLRM), and if $i=$ $1, \ldots, p$, it defines the Vector Autoregressive Model (VARM). The linear combination of MLRM and VARM produces MARDLM as derived. Assuming $\epsilon_{j t}$ is not identically and independently distributed with zero mean and constant variance, it describes the autocorrelation of the residual term. Therefore, the component of the error term assumed to follow the moving average process needs to be filtered out, leaving an uncorrelated residual of 
the model. This justifies an extension of the Multivariate Autoregressive Distributed Lag Model with additional components.

From (7), we have

$$
\begin{array}{llllr}
X_{j t}=C_{j}+\sum_{i=0, i=1}^{p} & \sum_{j=1}^{m} & \sum_{k=1}^{n} & \alpha_{i . j k} X_{k t-i}+ \\
\sum_{s=1}^{q} & \varphi_{s} \epsilon_{j t-s} & &
\end{array}
$$

where $\epsilon_{j t-s}$ is the $s$ lag moving average component of the error term in MARDLM with the parameter $\varphi_{s}$. The additional moving average component for the autocorrelated error term modifies (Usoro, 2019).

In this paper, we consider Multivariate Autoregressive Distributed Lag Moving Average Models for modelling Total Cases, New Cases, Active Cases, Discharged Cases and Total Deaths of the COVID-19 pandemic, where each response variable is a linear combination of the predictor variables at time $t$ and both response and predictor variables at time $(t-k)$. The suitability of Usoro (2019) will be investigated using the error term of each response model. The justification of the additional component to the MARDLM is based on the behaviour of each error term in the MARDLM.

\section{ANALYSIS AND RESULTS}

\section{Graphical presentations of COVID-19 Cases}

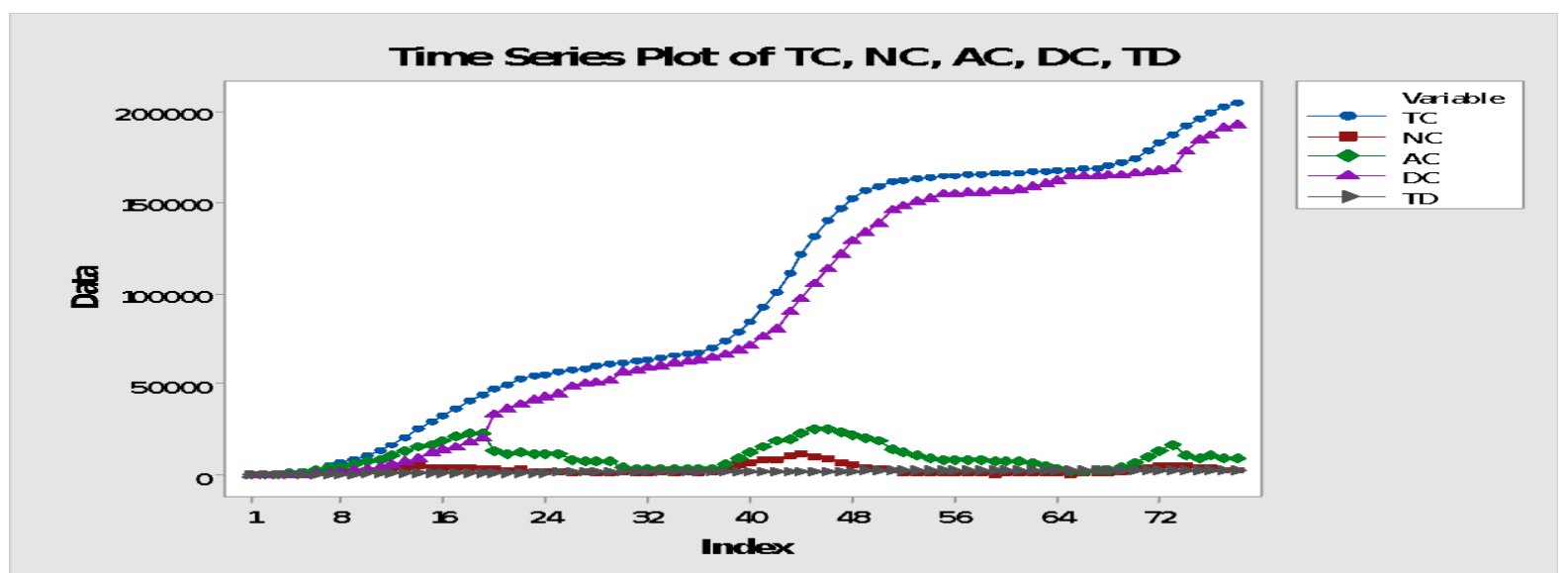

Figure 1: Multiple Time Graphs of All Reported Cases

Figure 1 is a multiple time plot of all cases, with total cases as the highest, followed by discharged cases, active cases, new cases, and total deaths as the least. From the graph, it is clear that the total and discharged cases compete favourably, which implies significant recovery of infected persons in comparison with the total cases. The weekly cumulative cases of COVID-19 at time added to the new cases at time gives the total cases at (where is the week-ended total confirmed cases)—Appendix 1. 


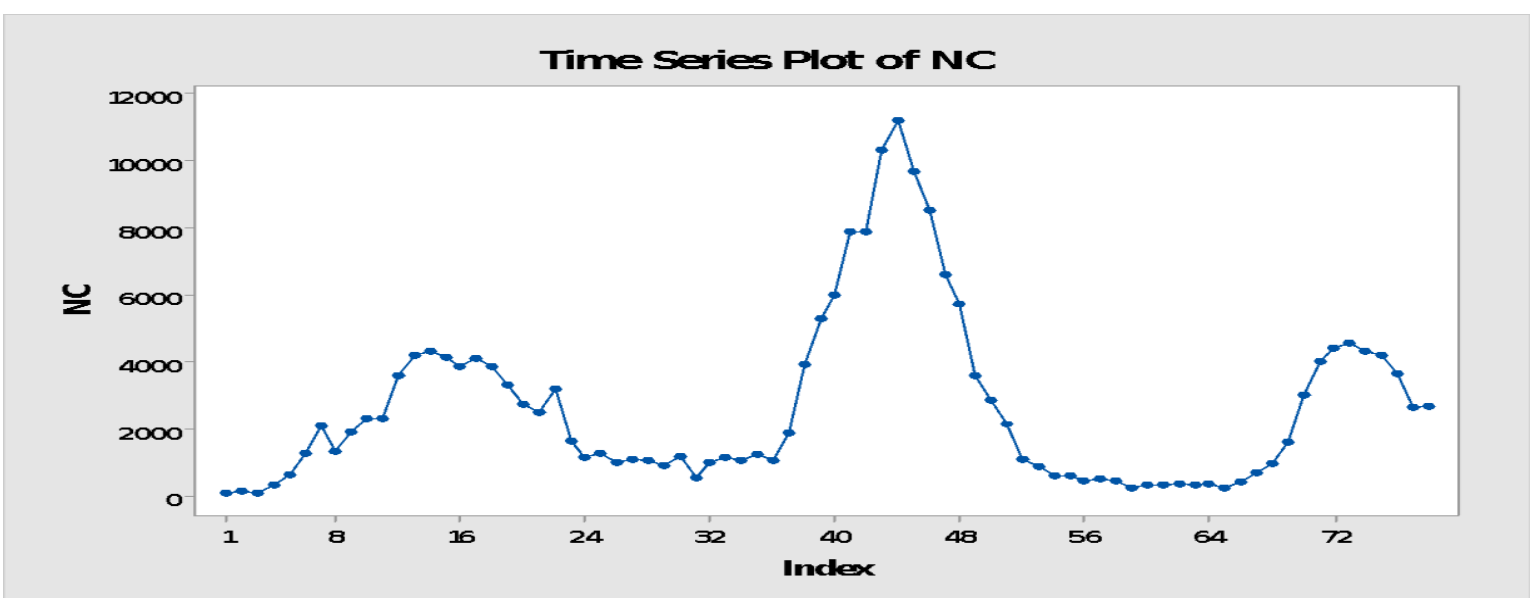

Figure 2: Time Plot of New Cases

Figure 2 presents three peaks from the COVID-19 pandemic in Nigeria. The first peak of the incidence after the discovery of the pandemic in March 2020 was within June and August 2020; the second peak was recorded between December 2020 and February 2021, being the second wave of the pandemic. The third wave of the scourge struck between July and the first week of September 2021. As shown in the graph, there is evidence of a decline in the last month i.e., in the 2nd, 3rd and 4th weeks of September 2021.

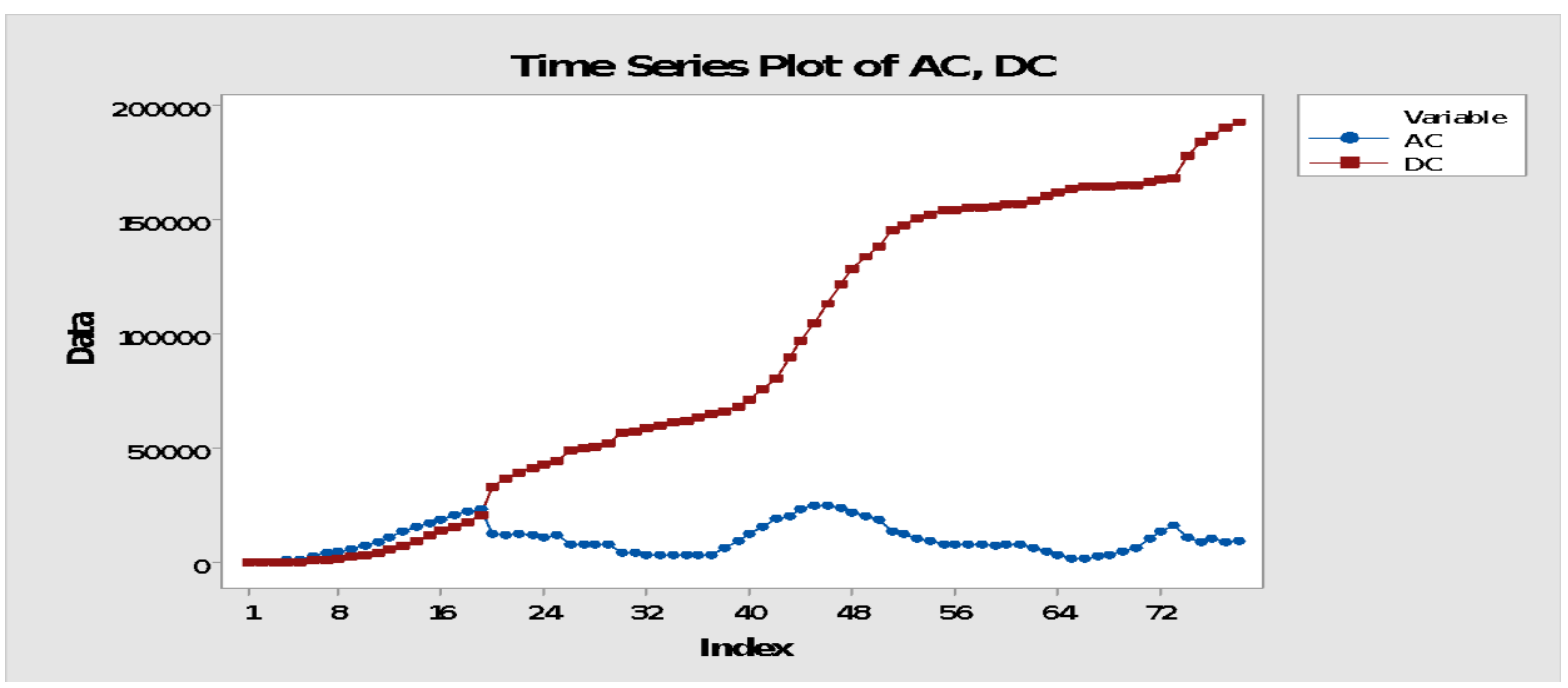

Figure 3: Time Plots of Active and Discharged Cases

Figure 3 presents the bivariate plots of active and discharged cases. There is a significant difference in the trends of the discharged cases versus active cases. The number of discharged cases increased with an increase in the number of active cases from August 3, 2020. 


\section{Analysis and Interpretation of Results}

This section looks at estimating the parameters of multivariate autoregressive distributed lag models with and without the error term's moving average process.

\section{Estimation of Parameters of MARDLM}

Table 1: Parameter Estimates of MARDLM

\begin{tabular}{|c|c|c|c|c|}
\hline Total Cases (TC) & Coefficient & SE. Coeff & T-Value & P-value \\
\hline Constant & 3.81 & 6.18 & 0.62 & 0.540 \\
\hline $\mathrm{NC}$ & 0.7888 & 0.0528 & 14.95 & 0.000 \\
\hline $\mathrm{AC}$ & 0.2091 & 0.0526 & 3.98 & 0.000 \\
\hline$\overline{\mathrm{DC}}$ & 0.2101 & 0.0525 & 4.0 & 0.000 \\
\hline TD & 0.2097 & 0.0585 & 3.59 & 0.001 \\
\hline TC-1 & 0.7968 & 0.0810 & 9.84 & 0.000 \\
\hline NC-1 & 0.00232 & 0.00365 & 0.64 & 0.527 \\
\hline$\overline{\mathrm{AC}-1}$ & -0.0067 & 0.0585 & -0.11 & 0.909 \\
\hline DC-1 & -0.0069 & 0.00584 & -0.12 & 0.907 \\
\hline TD-1 & -0.0062 & 0.0628 & -0.10 & 0.921 \\
\hline$\epsilon_{T C t-1}$ & -0.2474 & 0.1111 & -2.23 & 0.029 \\
\hline New Cases (NC) & Coefficient & $\overline{\text { SE. Coeff }}$ & T-Value & $\overline{\text { P-value }}$ \\
\hline Constant & -5.16 & 6.86 & -0.75 & 0.455 \\
\hline $\mathrm{TC}$ & 0.9755 & 0.0652 & 14.95 & 0.000 \\
\hline $\mathrm{AC}$ & 0.0256 & 0.0650 & 0.39 & 0.695 \\
\hline $\mathrm{DC}$ & 0.0243 & 0.0650 & 0.37 & 0.710 \\
\hline TD & 0.0296 & 0.0709 & 0.42 & 0.678 \\
\hline TC-1 & -1.0220 & 0.0651 & -15.70 & 0.000 \\
\hline NC-1 & -0.00024 & 0.00408 & -0.06 & 0.952 \\
\hline$\overline{A C}-1$ & 0.0215 & 0.0650 & 0.33 & 0.742 \\
\hline DC-1 & 0.0221 & 0.0649 & 0.34 & 0.734 \\
\hline TD-1 & 0.0222 & 0.0698 & 0.32 & 0.752 \\
\hline$\epsilon_{N C t-1}$ & -0.3237 & 0.1085 & -2.98 & 0.004 \\
\hline Active Cases (AC) & Coefficient & SE. Coeff & T-Value & P-value \\
\hline Constant & 2.6 & 12.9 & 0.20 & 0.838 \\
\hline TC & 0.912 & 0.230 & 3.98 & 0.000 \\
\hline $\mathrm{NC}$ & 0.090 & 0.229 & 0.39 & 0.695 \\
\hline $\mathrm{DC}$ & -0.99966 & 0.00256 & -390.52 & 0.000 \\
\hline TD & -1.0174 & 0.0484 & -21.04 & 0.000 \\
\hline TC-1 & 0.135 & 0.264 & 0.51 & 0.612 \\
\hline$\overline{\mathrm{NC}}-1$ & -0.00626 & 0.00762 & -0.82 & 0.414 \\
\hline $\mathrm{AC}-1$ & -0.046 & 0.122 & -0.38 & 0.708 \\
\hline
\end{tabular}




\begin{tabular}{|c|c|c|c|c|}
\hline DC-1 & -0.047 & 0.122 & -0.39 & 0.700 \\
\hline TD-1 & -0.047 & 0.131 & -0.36 & 0.723 \\
\hline Discharged Cases (DC) & Coefficient & SE. Coeff & T-Value & P-value \\
\hline Constant & 2.2 & 12.9 & 0.17 & 0.866 \\
\hline $\mathrm{TC}$ & 0.917 & 0.229 & 4.00 & 0.000 \\
\hline $\mathrm{NC}$ & 0.086 & 0.229 & 0.37 & 0.710 \\
\hline $\mathrm{AC}$ & -0.999 & 0.00256 & -390.52 & 0.000 \\
\hline TD & -1.0163 & 0.0487 & -20.85 & 0.000 \\
\hline TC-1 & 0.132 & 0.264 & 0.50 & 0.619 \\
\hline NC-1 & -0.00621 & 0.00762 & -0.82 & 0.418 \\
\hline AC-1 & -0.048 & 0.122 & -0.39 & 0.696 \\
\hline DC-1 & -0.049 & 0.122 & -0.40 & 0.690 \\
\hline TD-1 & -0.049 & 0.131 & -0.38 & 0.708 \\
\hline Total Deaths (TD) & Coefficient & SE. Coeff & T-Value & P-value \\
\hline Constant & 13.0 & 11.8 & 1.10 & 0.274 \\
\hline TC & 0.768 & 0.214 & 3.59 & 0.001 \\
\hline $\mathrm{NC}$ & 0.087 & 0.210 & 0.42 & 0.678 \\
\hline $\mathrm{AC}$ & -0.8537 & 0.0406 & -21.04 & 0.000 \\
\hline $\mathrm{DC}$ & -0.8525 & 0.0409 & -20.85 & 0.000 \\
\hline TC-1 & 0.136 & 0242 & 0.56 & 0.576 \\
\hline $\mathrm{NC}-1$ & -0.00465 & 0.00699 & -0.67 & 0.508 \\
\hline AC-1 & -0.048 & 0.112 & -0.43 & 0.666 \\
\hline DC-1 & -0.050 & 0.118 & -0.45 & 0.653 \\
\hline TD-1 & 0.054 & 0.120 & 0.45 & 0.655 \\
\hline
\end{tabular}

From Table 1, MARDLM with and without moving average components of the error terms were estimated. In each of the response variable models, some parameter estimates are significant while some are not, including positive and negative coefficients. The results revealed significant contributions of New Cases (NC), Active Cases (AC), Discharged Cases (DC) and Total Deaths (TD) to the Total Cases (TC). As a time series variable, one time lag of the Tocal Cases is significant. MA(1) model is fitted to the error term to capture the presence of the moving average component in the model. The present and the preceding Total Cases of COVID-19 have an impact on the New Cases as shown in the second set of the model estimates. The autocorrelation structure of the error term justifies the inclusion of MA(1) model to capture the moving average component of the model. As evident in Figures 1 and 3, an increase in the current time of the Discharged Cases and Total Deaths decreases the Active Cases. This explains the negative coefficients of the Discharged and Total Deaths in the model of the Active Cases. Apart from the Total Cases which has significantly contributed to every response variable, evidence has it that Active Cases and Total Deaths have negative impact on the Discharged Cases. This implies an increase in the Active Cases and Total Deaths decreases the number of the Discharged Cases and vice versa. Finally, Active and Discharged Cases have a 
strong negative impact on the Total Deaths, which accounts for the decrease in the Total Deaths as Active and Discharged Cases increase.

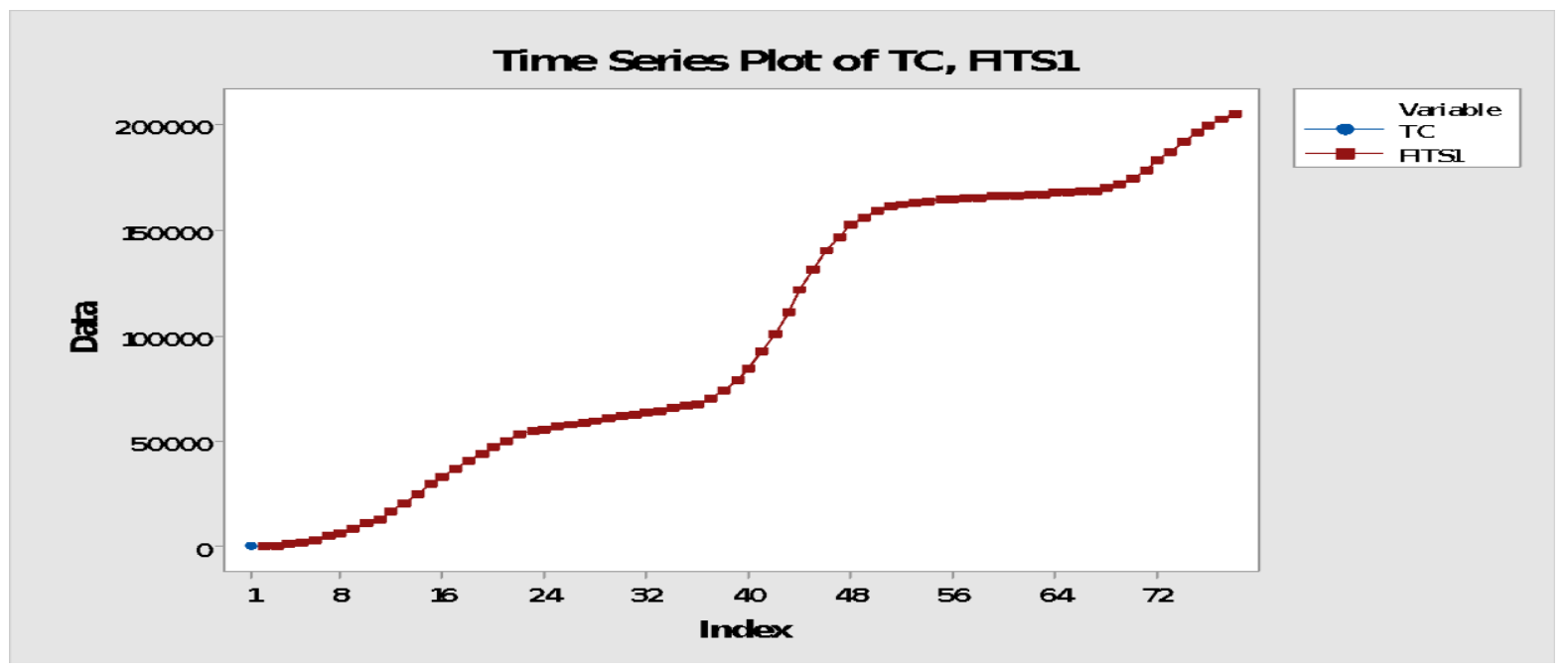

Figure 4A: Time Plots of Actual and Estimates of Total COVID-19 Cases

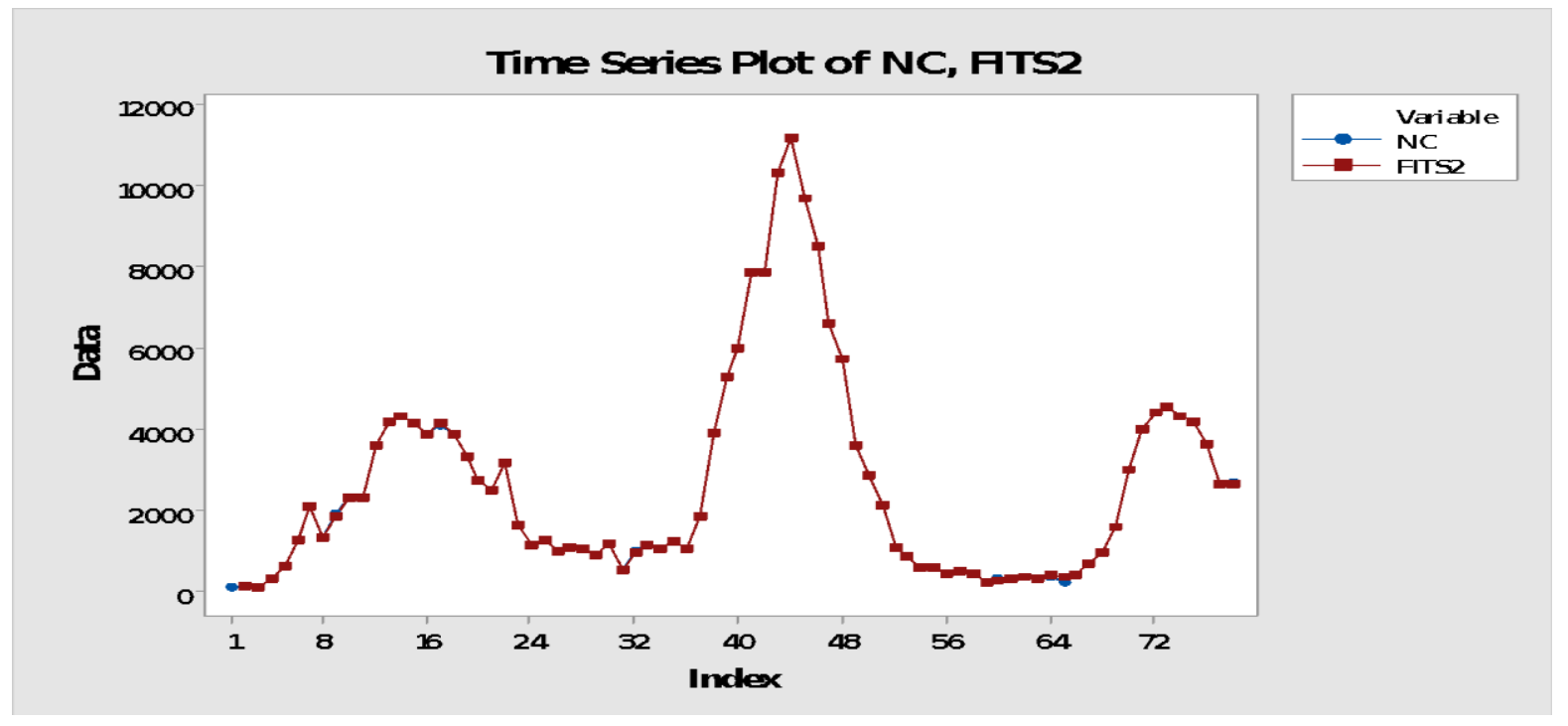

Figure 4B: Time Plots of Actual and Estimates of New COVID-19 Cases 


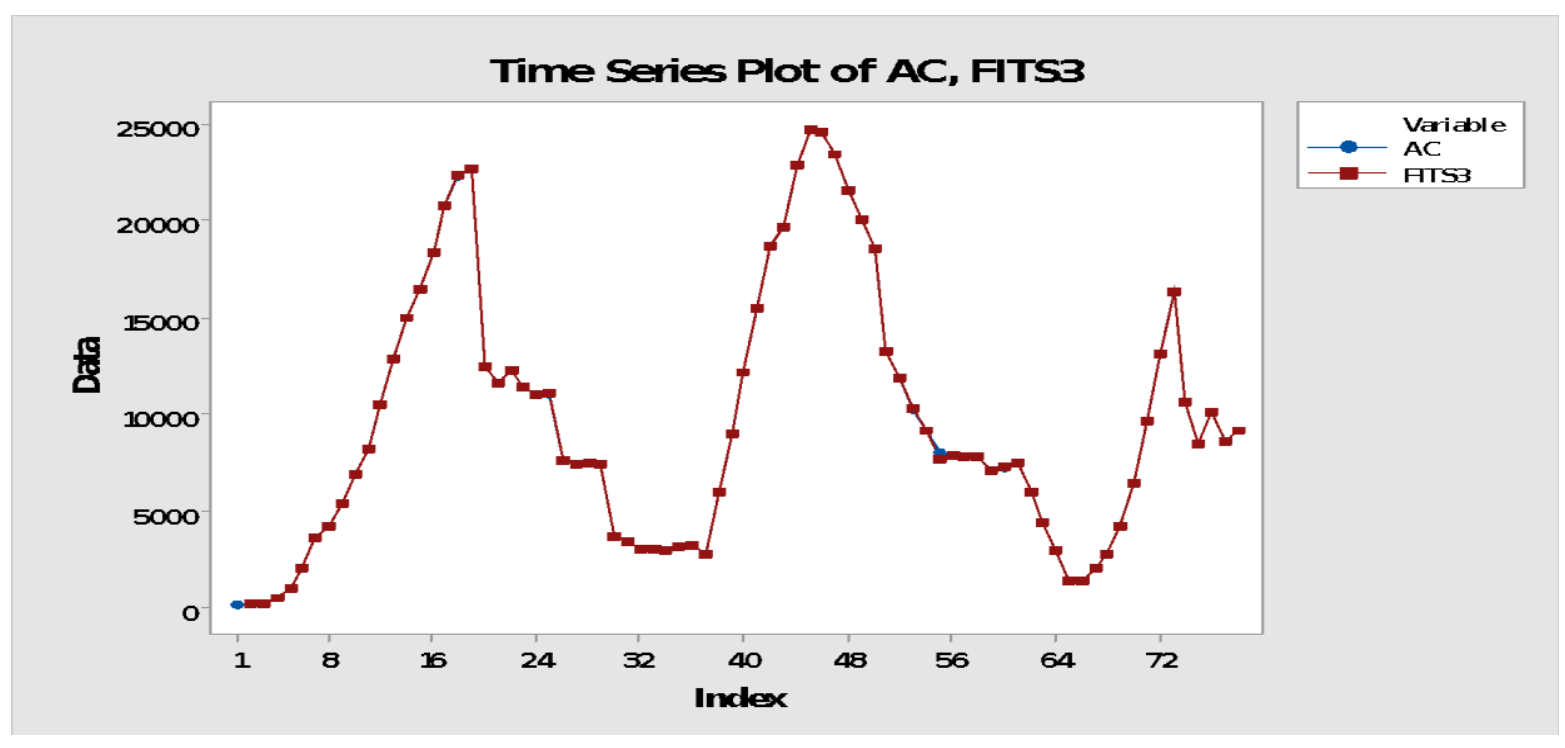

Figure 4C: Time Plots of Actual and Estimates of Active COVID-19 Cases

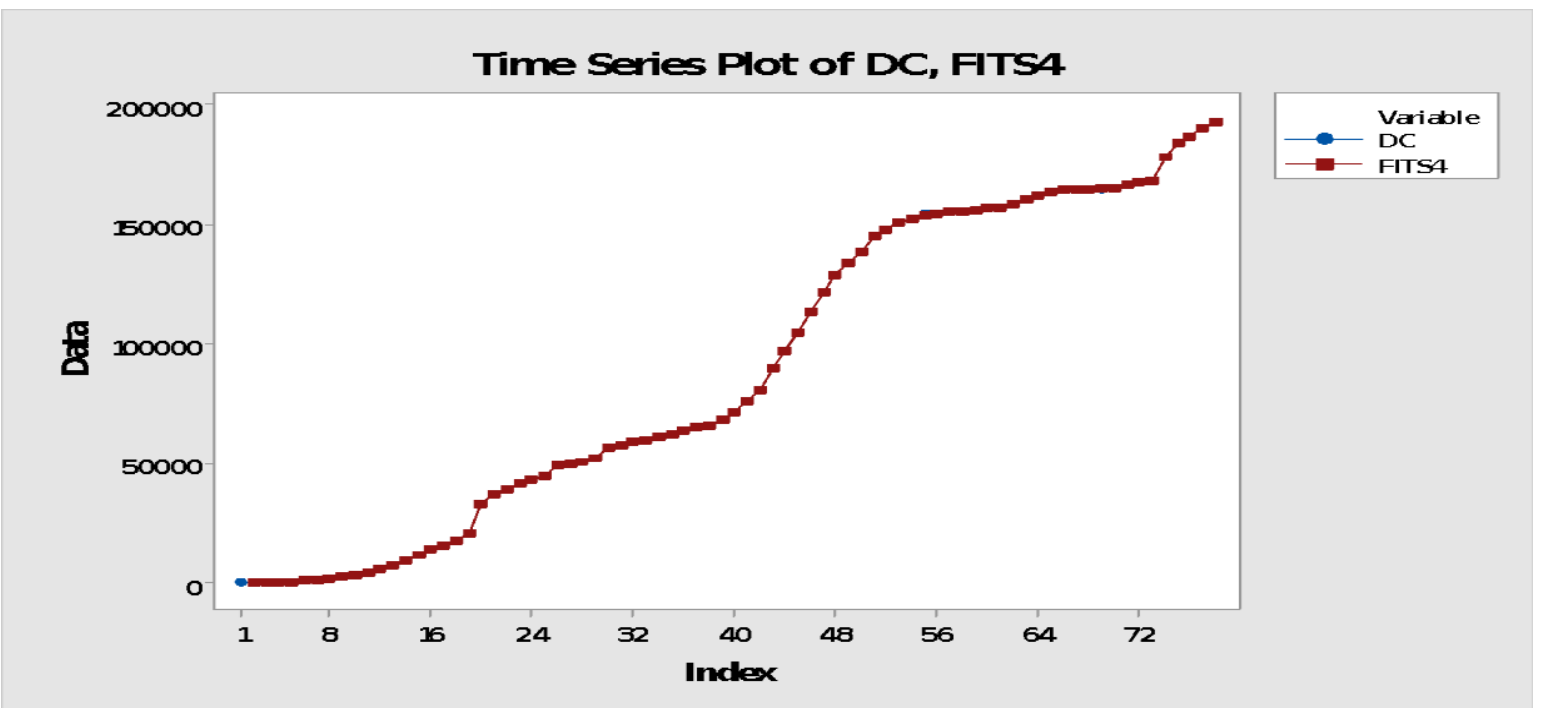

Figure 4D: Time Plots of Actual and Estimates of Discharged COVID-19 Cases 


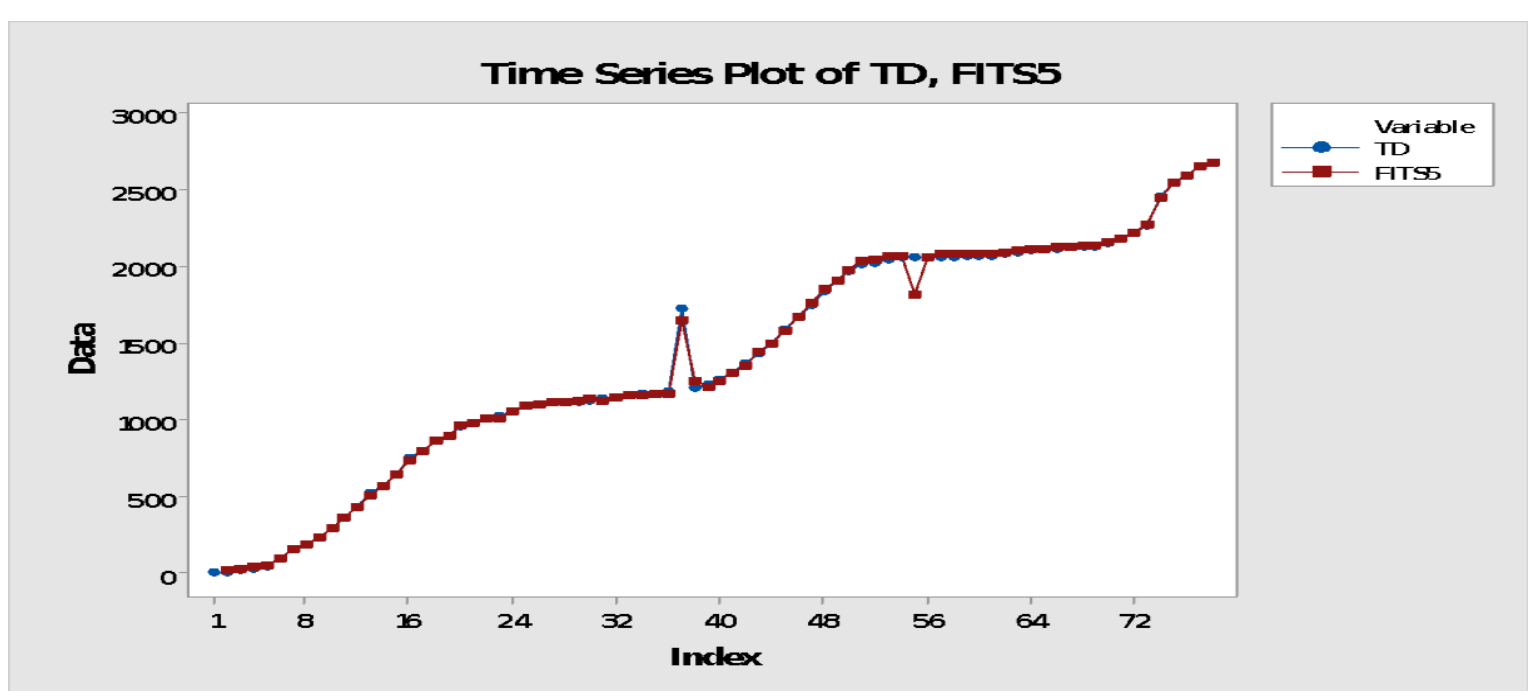

Figure 4E: Time Plots of Actual and Estimates of Total COVID-19 Cases of Deaths

The time plots of actual and estimated cases of the COVID-19 pandemic in Figures 4A-4E reveal suitability of the estimated MARDL-MA Models in fitting different cases of the pandemic in Nigeria. Appendix 1 contains the actual and estimated weekly data of COVID-19 Cases in Nigeria beginning from April 5, 2020 to September 26, 2021. The plots of the actual and estimates compete favourably as shown in the figures. The findings affirm MARDLM models for Active, Discharged and Total Deaths, while MARDL-MA Models have an advantage over MARDL Models for the estimation of Total and New Cases of COVID-19 in Nigeria.

\section{DISCUSSION AND CONCLUSION}

The emergence of the COVID-19 disease in almost all the nations in the world has been very threatening in view of its vulnerabilities in humanitarian settings, therefore subjecting the entire human race to distress and calamity, before the global enlightenment and awareness on possible means of transmission and non-pharmaceutical interventions as strategies to control the spread of the dreaded disease in the world. The daily mortality reports at the initial discovery of the pandemic in continents such as Europe, Asia and America were very devastating, overwhelming, and constituted serious panic as many nations mostly affected by COVID-19 appeared to have been overpowered by the pandemic. The effects of the novel coronavirus, which included human deaths and total closure of the world economy triggered global concern, thereby posing huge burdens on the economy of many nations in the era. In a bid to curb the spread of the disease in Nigeria, governments at all levels exhibited proactive measures, especially in the area of public enlightenment, creation of awareness and educating the human population on the possible ways of contracting the disease and the non-pharmaceutical measures to prevent and control the spread of the disease, while advocating for epidemiological investigations and clinical research to finding solution to the world health challenge. Governments at the state and federal levels have channelled huge resources in the fight against the disease in Nigeria. This is evidenced in the construction of special health facilities across 
many states in the country, building and strengthening the capacity of the existing tertiary health facilities, some of which serve as isolation centres for the COVID-19 infected persons. The good news about the prevalence of the novel COVID-19 is that, while many countries in the world today are engaged in clinical investigations and medical research to proffer solution to the life threatening pandemic, the humanitarian settings in Nigeria are very much acquainted with the trendy health challenges through governmental and non-governmental organisations to properly educate the public about the disease, as much as illuminating citizens on how to contain the coronavirus pandemic in the country.

Today, many nations in the world have built up capacity to overcome the scourge through extensive enlightenment campaigns, medical research and procurement of vaccines against the global pandemic. Despite the resurgence of the global pandemic in July 2021 as the third wave of the disease, the efforts of Nigerian government at all levels are reputed to have plausible impacts, as the country is experiencing a gradual decrease in the active and dead cases, while observing a significant increase in the daily discharged cases in September 2021. In spite of the pharmaceutical and non-pharmaceutical trials to fight against the disease, the need to have statistics view of the COVID-19 pandemic in Nigeria became pertinent in the sense that statistical research on the disease reveals the patterns of different categories of the lifethreatening pandemic with a view to assess and appraise the effects of government, nongovernmental organisations and other major stakeholders in the war against the dreaded disease. Sequel to the recent plausible development and the society's hope for a gradual extinction of the pandemic, it is encouraging to advocate more capacity building in the health sector, and strengthen the synergy between the private and public health organisations as a panacea for the global health challenge in our society. Statistically, Multivariate Autoregressive Distributed Lag Models (MARDLM) have been applied, and are found suitable for modelling and estimation of Active Cases, Discharged Cases and Total Deaths, while the modified model, MARDL-MA Model, is the best for Total and New Cases. Further findings from the research have it that every category of the COVID-19 pandemic, be it total cases, new cases, active cases, discharged cases or deaths, is dependent on the newly confirmed cases. Therefore, the COVID-19 pandemic will gradually go to extinction as the number of discharged cases increases with a drastic reduction in community transmission in Nigeria.

\section{REFERENCES}

Bollerslev, T., Engle, R. F. and Wooldridge, J. M. (1988): A Capital asset pricing model with time-varying covariances; The Journal of Political Economy, 96, 116-131.

Brown, Robert G. (1956): Exponential Smoothing for Predicting Demand. Cambridge, Massachuetts.

De livera, Alysha M. (2010): “Automatic Forecasting with a modified exponential smoothing state space framework". Department of Econometrics and Business Statistics, Monash University, Working Papers 10, No.10.

Diego, J. Pedregal (2019): Time Series analysis and forecasting with ECOTOOL. PLOS ONE, 14(10).

Enahoro A. Iboi, Oluwaseun O. Sharomi, Calistus N. Ngonghala and Abba B. Gumel (2020): Mathematical Modelling and Analysis of Covid19 Pandemic in Nigeria. MedRxiv

Engle, R. F. and Kroner K. F. (1995): Multivariate Simultaneous Generalised ARCH. Econometric Theory, 11, 122-150. 
Holt. C. C. (2004): Forecasting seasonal and trends by exponentially weighted moving averages. International Journal of Forecasting, Vol.20, No.1: 5-10.

Iram Naim, Tripti Mahara \& Ashraf Rahman Idrisi (2018): Effective Short-Term Forecasting for Daily Time Series with Complex Seasonal Patterns. International Conference on Computational Intelligence and Data Science. Procedia Computer Science, 132: 1832-1841.

Kayode Ayinde, Adewale F. Lukman, Rauf I. Rauf, Olusegun O. Alabi, Charles E. Okon and Opeyemi E. Ayinde (2020): Elsevier Public Health Emergency Collection. DOI: 10.1016/j.chaos.2020.109911.

Lingling Zhou, Ping Zhao, Dongdong Wu, Cheng Cheng \& Hao Huang (2018): Time Series model for forecasting the number of new admission inpatients. BMC Medical Informatics and Decision Making, 18, 39.

Maclean, Ruth and Abdi Latif Dahir, (Febryary 28, 2020): Nigeria Responds to First Coronavirus Case in Sub-Saharan African. The New Work Times. Retrieved March $10,2020$.

Oladeinde Olawoyin, (January 29, 2020): Coronavirus: Nigeria Strengthen Surveillance at five international airports. Premium Times. Retrieved March 10,2020.

Onyebuchi Ezigbo and Martins Ifijeh, (Febryary 1, 2020): Coronavirus Spread: World Health Organization lists Nigeria among high risk countries. This Day News Paper. Retrieved March 10,2020.

Premium Times (August 19, 2020).

Thomas Jaki (April 6, 2020): Positives in first published clinical trial of Covid-19 treatment. Science Daily.

Usoro, A. E. (2019): Modelling Passengers Traffic Status of Obong Victor Attah International Airport, Uyo, AKS. World Journal of Applied Science and Technology, Vol.11, No.1, pp 25-44.

Vini Yves Bernadin LOYARA, Remi Guillaume BAGRE and Frédric BERE (2020): Estimated of Covid-19 Sampling Mean in Burkina Faso. Journal of Mathematics Research; Vol.12, No.4, pp25-39.

Vasilis Marmarelis (2020): Predictive modelling of Covid-19 data in the US: Adaptive PhaseSpace approach, Volume 1: IEEE Open Journal of Engineering in Medicine and Biology. DOI:10.1109/OJEMB.2020.3008313. 


\section{APPENDIX}

\section{Appendix 1: Actual and Estimated Weekly Covid 19 Data From March 2020 to} September 2021.

\begin{tabular}{|c|c|c|c|c|c|c|c|c|c|c|}
\hline $\mathbf{S} / \mathbf{N}$ & TC & $\mathrm{NC}$ & $\mathbf{A C}$ & DC & TD & $\begin{array}{c}\text { EST(T } \\
\text { C) }\end{array}$ & $\begin{array}{c}\text { EST(N } \\
\text { C) }\end{array}$ & $\begin{array}{c}\text { EST(A } \\
\text { C) }\end{array}$ & $\begin{array}{c}\text { EST(D } \\
\text { C) }\end{array}$ & $\begin{array}{c}\text { EST(T } \\
\text { D) }\end{array}$ \\
\hline 1 & 111 & 69 & 107 & 3 & 1 & & & & & \\
\hline 2 & 232 & 121 & 194 & 33 & 5 & 235.66 & 115.988 & 196.6 & 35.158 & 17.4096 \\
\hline 3 & 323 & 91 & 228 & 85 & 10 & 326.8 & 85.9861 & 230.23 & 86.788 & 21.9713 \\
\hline 4 & 627 & 304 & 436 & 170 & 21 & 630.3 & 299.235 & 438.87 & 172.44 & 32.4777 \\
\hline 5 & 1273 & 646 & 994 & 239 & 40 & 1275.9 & 641.755 & 996.41 & 241.06 & 49.7329 \\
\hline 6 & 2551 & 1278 & 2071 & 393 & 87 & 2553.1 & 1274.73 & 2072.9 & 394.66 & 92.2102 \\
\hline 7 & 4641 & 2090 & 3587 & 902 & 152 & 4642.4 & 2087.86 & 3587.5 & 902.35 & 153.012 \\
\hline 8 & 5959 & 1318 & 4183 & 1594 & 182 & 5962.8 & 1315.61 & 4177.9 & 1588.7 & 183.052 \\
\hline 9 & 7839 & 1880 & 5350 & 2263 & 226 & 7839.4 & 1878.88 & 5351.4 & 2264.3 & 229.441 \\
\hline 10 & 10162 & 2323 & 6868 & 3007 & & 10162 & 2322.98 & & 3006.8 & 286. \\
\hline 11 & 12486 & 2324 & 8173 & 3959 & 354 & 12486 & 2324.72 & 8170.8 & 3957.1 & 350.663 \\
\hline 12 & 16085 & 3599 & 10445 & 5220 & 420 & 16082 & 3601.61 & 10447 & 5222.5 & 418.777 \\
\hline 13 & & 4159 & & & & 20241 & 4162.97 & & 6876.7 & \\
\hline 14 & 24567 & 4323 & 14995 & 9007 & 565 & 24564 & 4327.86 & 14992 & 9004.5 & 561.597 \\
\hline 15 & 28711 & 4144 & 16401 & 11665 & 645 & 28707 & 4149.29 & 16398 & 11663 & 638.586 \\
\hline 16 & & & & & & & & & & \\
\hline 17 & 36663 & 4105 & 20769 & 15105 & 789 & 36655 & 4113.87 & 20772 & 15109 & 788.005 \\
\hline 18 & 40532 & 3869 & 22300 & 17374 & 858 & 40524 & 3878.27 & 22303 & 17378 & 855.499 \\
\hline 19 & & & & & & & 3317.7 & & 20314 & 892 \\
\hline 20 & 46577 & 2736 & 12446 & 33186 & 945 & 46578 & 2731.44 & 12456 & 33193 & 959.225 \\
\hline 21 & 49068 & 2491 & 11596 & 36497 & 975 & 49067 & 2491.92 & 11596 & 36497 & 973.945 \\
\hline 22 & & & & & & 52224 & 3161.12 & & 38948 & 1001.82 \\
\hline 23 & & 1638 & & & & 53867 & 1638.42 & & 41509 & 1010.87 \\
\hline 24 & 55005 & 1140 & 10935 & 43013 & 1057 & 55004 & 1141.06 & 10937 & 43016 & 1054.59 \\
\hline 25 & & 1251 & & & & 56253 & 1252.53 & & 44158 & 1082.78 \\
\hline 26 & & 986 & 7575 & & & 57243 & 982.988 & & 48573 & 1102.46 \\
\hline 27 & 58324 & 1082 & 7422 & 49794 & 1108 & 58324 & 1080.98 & 7424.6 & 49797 & 1107.68 \\
\hline 28 & & 1021 & 7464 & & & 59346 & 1020.03 & & 50770 & 1112.57 \\
\hline 29 & 60266 & 921 & 7416 & & & 60267 & \begin{tabular}{|r}
919.89 \\
\end{tabular} & & 51737 & 1115.48 \\
\hline 30 & 61440 & 1174 & 3704 & 56611 & 1125 & 61444 & 1168 & 3708.9 & 56614 & 1129.89 \\
\hline 31 & 61992 & 552 & & & & 61997 & 547.976 & & 57462 & 1125.56 \\
\hline 32 & 62964 & 972 & 3028 & 58790 & 1146 & 62967 & 967.76 & 3029.6 & 58791 & 1143.83 \\
\hline 33 & 64090 & 1126 & 3026 & 59910 & 1154 & 64094 & 1121.79 & 3025.1 & 59909 & 1150.52 \\
\hline 34 & 65148 & 1058 & 2912 & 61073 & 1163 & 65152 & 1053.55 & 2910.1 & 61071 & 1158.84 \\
\hline 35 & 66383 & 1235 & 3140 & 62076 & 1167 & 66387 & \begin{tabular}{|l}
1230.8 \\
\end{tabular} & 3139 & 62075 & 1164.04 \\
\hline 36 & 67412 & 1029 & 3184 & 63055 & 1173 & 67417 & 1024.62 & 3181.8 & 63053 & 1169.25 \\
\hline 37 & 69255 & 1843 & 2755 & 64774 & 1726 & 69258 & 1840.69 & 2747.2 & 64766 & 1645.62 \\
\hline
\end{tabular}


African Journal of Mathematics and Statistics Studies

ISSN: 2689-5323

Volume 4, Issue 3, 2021 (pp. 118-134)

www.abjournals.org

\begin{tabular}{|c|c|c|c|c|c|c|c|c|c|c|}
\hline 38 & 73173 & 3918 & 5886 & 66090 & 1197 & 73173 & 3916.32 & 5888 & 66092 & 1250.78 \\
\hline 39 & 78434 & 5261 & 8910 & 68303 & 1221 & 78435 & 5260.09 & 8906.4 & 68299 & 1215.2 \\
\hline 40 & 84414 & 5980 & 12126 & 71034 & 1254 & 84415 & 5980.44 & 12120 & 71028 & 1246.97 \\
\hline 41 & 92251 & 7837 & 15413 & 75532 & 1306 & 92249 & 7838.65 & 15412 & 75531 & 1302.58 \\
\hline 42 & 100087 & 7836 & 18699 & 80030 & 1358 & 100087 & 7838.82 & 18690 & 80022 & 1350.91 \\
\hline 43 & 110387 & 10300 & 19635 & 89317 & 1435 & 110384 & 10300.9 & 19638 & 89319 & 1438.56 \\
\hline 44 & 121566 & 11179 & 22834 & 97228 & 1504 & 121565 & 11181.9 & 22826 & 97220 & 1500.06 \\
\hline 45 & 131242 & 9676 & 24667 & 104989 & 1586 & 131244 & 9678.66 & 24654 & 104976 & 1579.37 \\
\hline 46 & 139748 & 8506 & 24556 & 113525 & 1667 & 139748 & 8507.43 & 24553 & 113521 & 1669.74 \\
\hline 47 & 146354 & 6606 & 23408 & 121193 & 1753 & 146355 & 6606.35 & 23407 & 121192 & 1757.23 \\
\hline 48 & 152074 & 5720 & 21567 & 128668 & 1839 & 1520 & 571 & 21574 & 28675 & .05 \\
\hline 49 & 155657 & 3583 & 20008 & 133742 & 1907 & 155658 & 3581.83 & 20013 & 133747 & 1915.18 \\
\hline 50 & 158535 & 2878 & 18511 & 138055 & 1969 & 158533 & 2876 & 18526 & 138070 & .45 \\
\hline 51 & 1606. & 2122 & 13245 & 145399 & 2013 & 160 & & 13261 & & \\
\hline 52 & 161737 & 1080 & 11808 & 147899 & 2030 & 161739 & 1074.76 & 11820 & 147911 & 4.44 \\
\hline 53 & 162593 & 856 & 10237 & 150308 & 2048 & 162594 & 849.927 & 10253 & 150324 & .13 \\
\hline 54 & & 602 & 9139 & & 2058 & & & 9154.4 & & \\
\hline 55 & 163793 & 598 & 7926 & 154107 & 2060 & 163858 & 598 & 7642.1 & 153823 & 1821.83 \\
\hline 56 & 164233 & 440 & 7840 & 154332 & 2061 & 164233 & 440 & 7840 & 154332 & 2061 \\
\hline 57 & & & 731 & & & & & & & \\
\hline 58 & 165181 & 462 & 7757 & 155361 & 2063 & 165183 & 455.316 & 7771.9 & 155376 & 2078.8 \\
\hline 59 & 165419 & 238 & 7057 & 156297 & 2065 & 165422 & 230.415 & 7071.7 & 156312 & 2081.18 \\
\hline 60 & & 290 & 7230 & & 2066 & & & & & \\
\hline 61 & 166019 & 310 & 7476 & 156476 & 2067 & 166021 & 303.274 & 7491.3 & 156492 & 2082.91 \\
\hline 62 & 166367 & 348 & 5946 & 158344 & 2077 & 166370 & 339.131 & 5962 & 158360 & 2093.93 \\
\hline 63 & & 320 & 4385 & & 2089 & & & 4399.1 & & .69 \\
\hline 64 & 167105 & 362 & 2929 & 162073 & 2103 & 167067 & 407.223 & 2936.9 & 162081 & 2110.93 \\
\hline 65 & 167467 & 209 & 1399 & 163949 & 2119 & 167353 & 350.207 & 1396.1 & 163946 & 2116.1 \\
\hline 66 & 167859 & 392 & 1356 & & 2121 & & 381.129 & 1366.1 & & 0.71 \\
\hline 67 & 168552 & 693 & 1989 & 164439 & 2124 & 168558 & 682.831 & 1998.6 & 164449 & 2132.86 \\
\hline 68 & 169518 & 966 & 2694 & 164697 & 2127 & 169524 & 956.141 & 2703.3 & 164706 & 2135.96 \\
\hline 69 & 171097 & 1579 & 4178 & 164787 & 2132 & & 1570.32 & 4188 & 164797 & 2141.25 \\
\hline 70 & 174088 & 2991 & 6399 & 165537 & 2152 & 174091 & 2983.68 & 6410.7 & 165549 & 2161.24 \\
\hline 71 & 178086 & 3998 & 9575 & 166328 & 2183 & 178088 & 3992.63 & 9583 & 166337 & 2188.55 \\
\hline 72 & 182503 & 4417 & 13152 & 167132 & 2219 & 182504 & 4413.67 & 13158 & 167139 & 2223.61 \\
\hline 73 & 187023 & 4520 & 16300 & 168455 & 2268 & 187022 & 4518.15 & 16308 & 168464 & 2273.68 \\
\hline 74 & 191345 & 4322 & 10613 & 178278 & 2454 & 191351 & 4311.27 & 10623 & 178286 & 2451.57 \\
\hline 75 & 195511 & 4166 & 8435 & 184524 & 2552 & 195518 & 4156.22 & 8439 & 184527 & 2547.89 \\
\hline 76 & 199151 & 3640 & 10140 & 186413 & 2598 & 199156 & 3633.82 & 10140 & 186414 & 2592.36 \\
\hline 77 & 201798 & 2647 & 8580 & 190563 & 2655 & 201805 & 2638.01 & 8583.3 & 190566 & 2652.67 \\
\hline 78 & 204456 & 2658 & 9157 & 192622 & 2677 & 204460 & 2650.83 & 9164.6 & 192630 & 2679.51 \\
\hline
\end{tabular}

